\title{
基于浮动车数据的旅游景点周边路网容量研究 以厦门岛为例
}

\author{
高悦尔, 陈舒婷, 郑承于,边经卫 \\ (华侨大学建筑学院, 福建 厦门 361021)
}

\begin{abstract}
摘要: 随着旅游业的快速发展, 旅游城市在法定节假日出现了交通需求的大幅增长, 导致不同程度的交通问题。本 文利用厦门市的浮动车数据 $(\mathrm{FCD})$, 分别选取 2014 年 10 月 1 日与 10 月 14 日作为节假日与工作日的样本进行研究, 首先通过 $\mathrm{OD}$ 点核密度估计判断节假日与工作日出行在时空上的差异, 确定研究的时空范围; 其次, 通过对 FCD 数 据进行核密度估计分析旅游景点周边的车流量变化, 并与路段的等级、车道数等道路设施属性进行对比, 推测路网 的容量 ; 最后进一步通过计算路网中路段行程车速验证方法的可行性。结果显示: 节假日期间的旅游交通与工作 日的通勤交通在时空分布上存在差异, 同时基于FCD数据研究旅游景点周边的路网容量是有效的, 厦大一南普陀 景点周边路网在节假日期间无法满足车流量的需求。本文对旅游景点周边路网容量的评估结果可为旅游旺季旅 游路网的合理规划与管控提供依据。
\end{abstract}

关键词: 旅游交通; 路网容量; 核密度估计法; 浮动车数据; 厦门岛

1 引言

随着中国旅游业的快速发展, 旅游城市在法定 节假日出现了交通量的大幅增长。旅游交通由于 旅游出行目的地(旅游景点)与出行时间(节假日)的 聚集, 造成一定时间内旅游交通需求大幅增长, 使 得在节假日期间旅游景点周边的路网面临巨大的 交通压力, 造成了超负荷的现象。2005-2014年, 厦 门市年接待游客从 1712.88 万人次增长到 5337.86 万人次, 因此, 针对节假日期间旅游景点周边骤增 的交通量带来的问题, 对城市旅游景点周边路网进 行研究, 可为厦门城市旅游交通规划与管理提供 依据。

国内外对城市路网的研究主要集中在路网容 量、路网结构、路网布局等方面。在路网容量方面, 学者们研究了不同道路条件下的路网容量。如冷 军强等(2010)以路段行程时间可靠性及容量为约束
条件,设计了用于评估冰雪条件下的路网容量可靠 性模型; 王建等(2013)构建了一种用于描述交叉口 多相位信号控制路网容量的双层规划模型, 通过信 号灯配时使路网容量达到最大。在对路网结构的 研究中, 大多数学者对城市内部路网结构的影响因 素进行分析, 提出路网结构的优化方法。如 Jenelius(2009)发现路网结构的脆弱性差异取决于交通 系统的基本属性和人口密度; 龙雪琴等(2012)考虑 城市交通事故与道路网结构的关系, 结合交通事故 导致的时间延误, 提出了基于交通安全的城市路网 结构优化方法。在路网布局方面, 孙慧娟(2010)运 用“点线面多模块层次分析”的方法, 对西安城市快 速路交通网络进行“点”“线”“面”3 个层次分析, 最 终提出了城市快速路交通网络布局规划原则。已 有对路网的研究通常是指行政区划下的城市路网, 没有特别针对某个交通问题严重的节点进行周边 路网的分析, 而对交通问题突出节点的研究主要集

收稿日期: 2016-06;修订日期: 2016-10。

基金项目: 国家自然科学基金项目(51608209); 华侨大学高层次人才科研启动项目(600005-Z15Y0035) [Foundation: National Natural Science Foundation of China, No.51608209; High Level Talent Research Project of Huaqiao University, No.600005-Z15Y0035]。

作者简介:高悦尔(1983-),女,福建晋江人,讲师,博士,从事城市土地利用与城市交通规划研究,E-mail: gaoyueer123@gmail.com。 
中在大型活动的人流车流运行特征方面, 同时提出 活动区域相应的交通组织措施。张伟(2014)设计了 一类大型公共场所的行人交通状态评价方法以及 行人交通应急疏散模型; 陈小鸿等(2013)基于车流 波动理论, 分析城市大型活动车队行驶特征及对交 通的影响。目前, 浮动车数据 (floating car data, FCD)在信息采集、数据处理等方面已形成一套较为 成熟的技术方法。通过对浮动车数据的处理, 能够 获取城市道路实时交通信息(如行程速度、行驶时 间等), 以反映城市路网的交通状态特征。刘伟铭 等(2011)以浮动车的平均行程速度和平均行车时间 延误作为道路状态评价指标, 建立模糊综合评价模 型对评价时段内路网交通状态的进行判别; $\mathrm{Li}$ 等 (2011)以浮动车数据为基础, 根据网络内核密度估 计(NKDE)和网络线密度估计(NLDE)2 种方式提取 信息点(POI)数据信息, 提出一个基于实时行程速度 的平日和周末的高峰时段、非高峰时段的时空动态 判别模型。由于 FCD 数据量大、覆盖范围全面的优 点, 近年来, 有学者开始用动态的 FCD 数据分析静 态的路网问题和交通的可达性问题。如马林兵等 (2015)将城市路网密度与路网中浮动车分布核密度 进行对比, 提出一种基于 FCD 数据的城市有效路网 密度评价方法; 胡继华等(2012)通过浮动车数据, 建 立出租车经验路径库, 计算广州市中心城区的可达 性; 陈建国等(2016)通过浮动车数据, 分析交通拥堵 对广州市中心城区急救医疗设施时空可达性的影 响。另外, 也有研究从其他动态数据源分析城市交 通问题(吴健生等, 2012)。

在旅游交通的出行需求持续增长、季节性特征 日益显著以及安全问题不断暴露的形势下, 本文以 厦门岛为例, 利用动态的 FCD 数据对旅游景点周边 的路网容量进行研究, 进一步发现静态路网存在的 问题, 旨在为城市旅游交通规划管理提供参考 依据。

\section{2 数据与方法}

\section{1 研究区域与数据来源}

厦门市位于中国东南沿海, 福建省南部, 辖思 明区、湖里区、集美区、海沧区、同安区、翔安区 6 个 区。本文主要研究范围为厦门岛, 包括思明区和湖 里区 2 个行政区(图 1)。区域内含厦门大学、鼓浪 屿、曾厝垵、南普陀寺、中山路步行街、环岛路等景

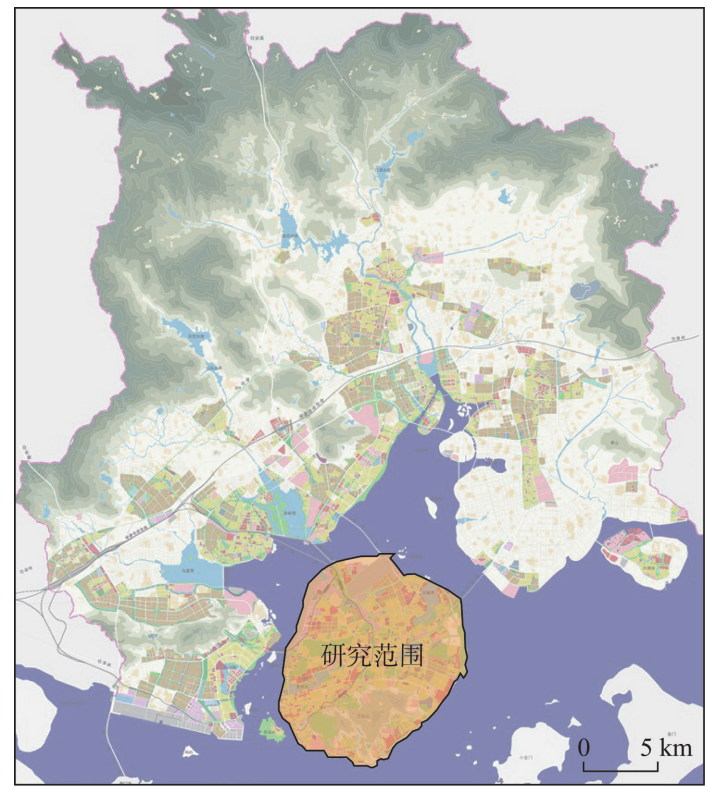

图 1 研究范围示意图

Fig.1 Spatial scope of the study area

点, 这些城市旅游景点节假日期间往往成为人流车 流高度聚集的节点。

目前, 厦门市浮动车系统拥有出租车 5000 余 辆, GPS 数据回传间隔为 $10 \sim 30 \mathrm{~s}$, 每天采集 GPS 数 据 8000 多万条, 数据内容包括车辆编号、当前日期、 当前时刻、经纬度坐标、瞬时车速、方向角、载客标 志等, 数据涵盖厦门市市域范围内高速公路、快速 路、主干路、次干路、支路共计 11696 个路段,覆盖率 达到 90\%。本文选取了法定节假日(2014年 10 月 1 日)与正常工作日 (2014 年 10 月 14 日)的 FCD 数据 展开研究。

\section{2 研究方法}

核密度估计法 (Kernel Density Estimation, $\mathrm{KDE})$ 是借助一个移动的单元格, 利用核函数来计 算单元格内要素密度的估计法(汤国安等, 2016)。 核密度估计法可将浮动车瞬时坐标与路网 2 种不同 的空间要素, 通过转化成相同的栅格数据进行分 析。将核密度多时段栅格数据进行对比研究, 能直 观地发现路网中浮动车流量的时空变化特征。

在 GIS 的核密度制图中, 落人搜索区内的点具 有不同的权重,靠近格网搜寻区域中心的点或线会 被赋以较大的权重, 随着其与格网中心距离的加 大, 权重降低。核密度估计在二次函数表达形式为 (Shi, 2010):

$$
f(x, y)=\frac{1}{n h^{2}} \sum_{i=1}^{n} k\left(\frac{d_{i}(x, y)}{h}\right)
$$


式中: $f(x, y)$ 为位于 $(x, y)$ 位置的密度估计; $n$ 为观测 数值; $h$ 为带宽或平滑指数; 核函数 $k(\cdot)$ 是一个权函 数; $d_{i}(x, y)$ 为 $(x, y)$ 位置距第 $i$ 个观测位置的距离。

城市道路网络容量是指在约束条件一定的情 况下,城市道路交通网络系统所能容纳或服务最大 车辆数(李晶等, 2006)。本文采用核密度估计法分 析 FCD数据在空间上的聚集特征,描述厦门节假日 期间游客旅游活动的时空特征, 通过出租车在单位 时间的密集程度并结合道路设施属性进一步对旅 游景点周边的路网容量进行评估。具体步骤如下:

(1) 研究时空范围的界定。旅游高峰时间的交 通状态极大部分是因游客的旅游活动引起的, 由于 旅游出行属于弹性出行, 对出租车的需求较高, 因 此旅游高峰时间的出租车运营 OD 点(“O”是 Origin 的缩写, 指出行的出发地点; “ $D$ ”是 Destination 的缩 写, 指出行的目的地)能较好地表达游客出行的高 峰小时与游客对旅游目的地的选择。首先对比节 假日与工作日的出租车载客次数, 选取与工作日出 租车载客次数差距较大的高峰小时, 确定为研究的 高峰时间; 将出租车运营 OD 点匹配至 GIS 电子地 图, 运用核密度估计法, 对高峰时间出租车运营 OD 点进行核密度分析, 然后选择其中一个景点核密度 高等级点作为研究对象, 并根据核密度等级的边缘 递减效应确定研究的路网范围, 从而得到旅游景点 周边路网的时空范围。

(2) 旅游景点周边路网车流量的空间分析。以 $5 \mathrm{~min}$ 为间隔, 将高峰小时的 FCD 数据分为 12 组, 分别匹配至 GIS 地图, 并进行核密度分析。根据核 密度的空间分布变化可以得到该高峰小时内景点 周边路网承担车流量的空间变化特征。

(3) 对比研究路网容量。通过核密度分析得到 车流量在路网中不均衡的空间分布, 将其与路段实 际道路设施属性进行对比, 推测目前旅游景点周边 路网存在的问题。最后, 通过运用FCD数据对研究 范围内路段行程车速进行计算,验证推测结果。

\section{3 节假日路网容量评估}

\section{1 旅游活动强度峰值的时空范围}

由于主要出行目的的不同, 节假日与工作日的 出行高峰时间与地点可能会存在差异, 通过对该差 异的分析, 能够减弱其他交通因素的干扰, 得到旅 游交通的高峰时间与空间范围。FCD 数据虽只包
含出租车数据, 但旅游景点及旅游基础设施周边常 常存在大量的出租车上下客现象, 同时从 FCD 数据 中提取的交通信息已成为反映城市道路交通状态 的重要手段之一, 因此本文使用 FCD 数据对节假日 期间的交通问题进行分析。利用出租车 GPS 数据, 从每辆出租车的第一条数据开始记录车辆的空间 位置与载客状态, 当载客状态发生变化时, 完成一 次记录, 得到出租车的 OD 点坐标与对应的时间信 息, 即一次上下客的坐标与时间信息。出租车的 OD数据能很好地体现节假日游客出行的目的地, 如景点的选择, 因此利用出租车上下客载客数据能 得到节假日游客旅游活动的聚集倾向, 从而得到旅 游活动高峰的空间分布。

\subsection{1 旅游活动强度高峰时间}

将出租车 OD数据按小时进行统计, 并绘制折 线图, 对比分析得到出租车运营高峰时间。本文分 别选取了 10 月 1 日和 10 月 14 日 7:00-19:00 的 FCD 数据, 将每小时的出租车 OD数据分别匹配至 GPS 地图,删除坐标异常(没有落到道路上的点)的上下 客记录点, 得到整个厦门岛正常运营的出租车上下 客记录点。

节假日(10月 1 日)7:00-19:00, 厦门岛内共记录 276850 条出租车上下客信息, 其中出现了 2 个高峰 小时, 分别为 $12: 00-13: 00$ 及 18:00-19:00。工作日 (10月 14 日)的 7:00-19:00, 厦门岛内共记录 260813 条出租车上下客信息。相比于节假日, 出租车当日 运营数量减少了 $5.8 \%$, 高峰时间也出现了明显的差 异。工作日(10月 14 日)出租车的运营高峰分别为 9:00-10:00 以及 $14: 00-15: 00$ (图 2)。通过对比节假 日与工作日每小时的出租车运营数量差值(图 3), 发 现 7:00-10:00 差值出现了负值, 说明该时间段的工

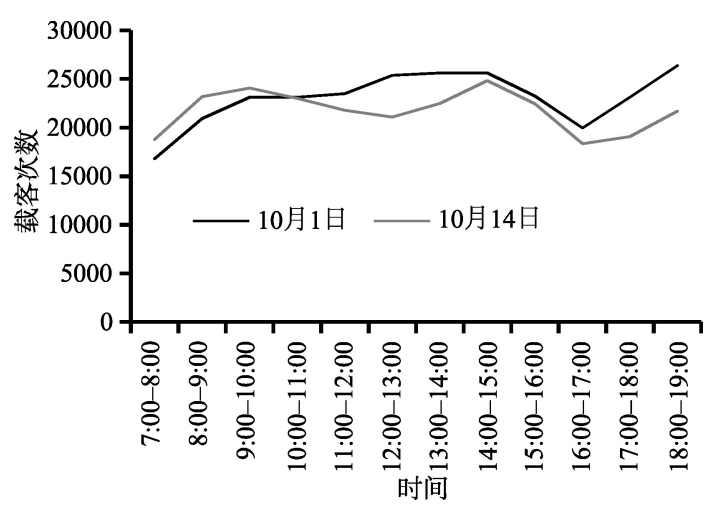

图2 10 月 1 日与 14 日出租车载客次数对比

Fig.2 Taxi passenger numbers of 1 October and 14 October 
作日出租车载客次数大于节假日出租车载客次数, 可认为该时间段为工作日的通勤时间; 并且在 10 月 1 日的该时间段,出租车载客次数较少,也是节假日 旅游出行的低峰时间。另外, 12:00-13:00与 18:0019:00 节假日出租车载客次数远大于工作日, 可认 为节假日这 2 个时间段以旅游为目的的出行活动有 了较大的增长。

利用核密度估计法进一步研究出租车运营记 录点在各个时间段的空间分布特征。本文选取了 工作日通勤高峰时间 7:00-8:00、节假日与工作日差 值较大的 12:00-13:00 与 18:00-19:00, 以研究不同时 间段工作日与节假日出租车运营 OD点的空间分布

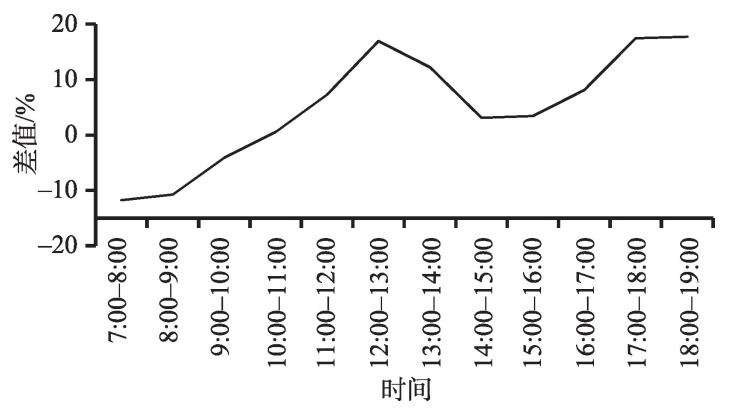

图310月 1 日与 14 日出租车载客次数差值

Fig.3 Difference of taxi passenger numbers between 1 October and 14 October
差异。分析中的搜索半径即核密度分析带宽, 经过 多次试验, 确定为 $900 \mathrm{~m}$, 与 GIS 软件自动生成的 $488 \mathrm{~m}$ 搜索半径对比发现,利用 $900 \mathrm{~m}$ 生成的密度 曲面较为平滑, 并能清晰地识别出浮动车分布的密 度中心。分析得到的核密度值分布在 0 1550000之 间,且核密度最大值出现在 10 月 1 日 12:00-13:00, 将该时间段的核密度值按照自然间断法分为等级 1 (密度极小)、等级 2 (密度小)、等级 3 (密度较小)、等 级 4 (密度较大)、等级 5 (密度大) 等 5 个级别, 并以此 分段数据区间为标准,运用于每个时间段的核密度 等级划分(表1)。通过分析, 可得出:

(1) 与工作日相比, 节假日出租车 OD点核密度 等级与分布范围有较大增量。对比发现, 10 月 1 日 7:00-8:00、12:00-13:00、18:00-19:00 等3个时间段的 高等级密度核的范围与最高值都远大于 14 日的 3 个时间段,说明节假日期间厦门岛的出租车交通量 在高密度核周围有一个较大的增量, 且影响范围也 有所扩大。

(2) 节假日与工作日出租车 OD 点核密度空间 分布具有差异性。对比 10 月 1 日与 10 月 14 日 3 个 时间段的核密度分布可以发现, 高核密度点的位置 和范围存在较大差异。工作日与节假日在核密度 等级 4 与等级 5 的位置均有了一定的偏移, 并且旅 游景点的核密度值有了明显的增加, 说明节假日交

表 1 厦门岛内出租车 OD 点核密度估计分布特征

Tab.1 Distribution characteristics of taxies' origin-destination (OD) points by Kernel density estimation (KDE) in Xiamen Island

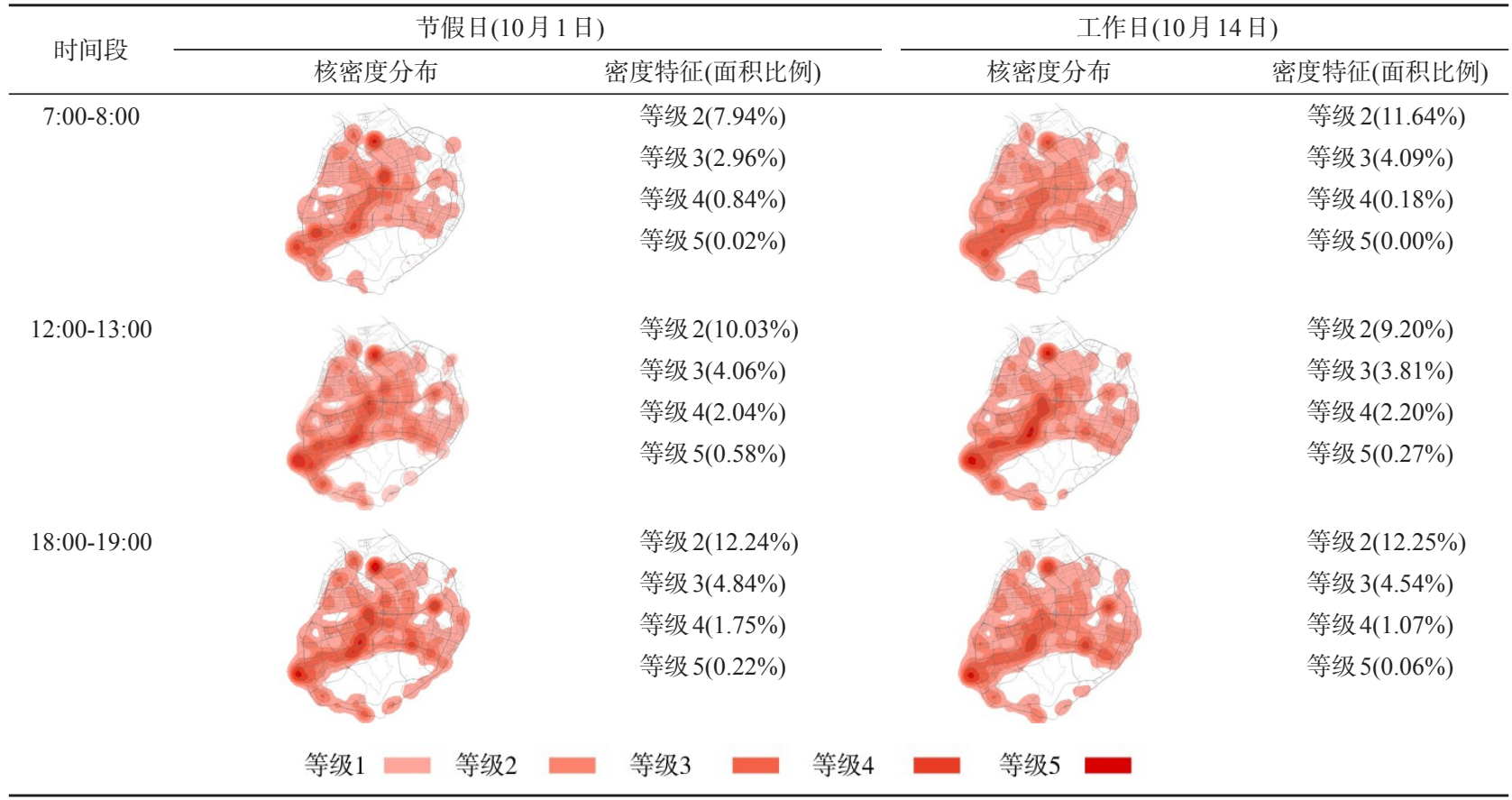


通压力大的节点与工作日不同, 所以应与工作日的 路网分开研究。

(3) 12:00-13:00 的出租车 OD 点核密度更大, 整 个岛内出租车运营数量增加最多的时间段是 18:0019:00。对比 7:00-8:00、12:00-13:00、18:00-19:00 这 3 个时段发现,核密度最大的点出现在 12:00-13:00, 而出租车增量最大的 18:00-19:00 密度分布更为均 匀。这说明 12:00-13:00 的旅游景点交通问题更为 突出。因此,将选取 10月 1日的 12:00-13:00这一时 间段的路网作为研究对象。

\subsection{2 旅游活动强度高峰空间}

提取旅游高峰时间 10月 1 日 12:00-13:00 的出 租车 OD数据, 并进行核密度分析。从表 2 可以看 出, 核密度增量较大的空间即为旅游活动高峰空 间, 均出现在景点附近, 如: 轮渡一中山路、厦大一 南普陀寺、胡里山炮台、曾厝垵等(图 4)。这证实了 节假日期间厦门岛内旅游景点周边路网压力骤增, 可能存在路网容量不足的情况, 因此需要对旅游景 点周边路网进一步分析。核密度最大值出现在轮
渡一中山路景点, 但由于轮渡一中山路附近的交通 更为复杂, 涉及水陆两大交通枢纽, 远不止包含旅 游活动所产生的交通问题, 因此本文选取节假日与 工作日在高峰小时内核密度差异较大的厦大一南 普陀寺景点进行周边路网分析。

对表 2 进一步分析发现, 厦大一南普陀寺景点 周围的核密度等级由等级 2 提高到等级 4 , 说明该 景点在旅游时间承担着比工作日更高的交通压 力。根据核密度等级的边缘效应, 成功大道以北核 密度开始上升, 说明其受到第一码头景点较大的影 响; 而厦大一南普陀寺景区以东核密度等级为 0 , 则 是由于该区域出租车无法通行造成。因此本文选 择演武大桥、成功大道与厦门大学北面山体围合的 区域作为研究对象(图 5), 对厦门大学一南普陀景点 周边旅游路网进行研究。

\section{2 旅游景点周边路网车流量分析}

根据上述旅游景点对周边路网影响的时空范 围, 通过在研究范围内对 FCD 数据进行核密度分 析, 利用分布在旅游景点周边路网上的 $\mathrm{FCD}$ 数据聚

表 2 节假日出租车 OD点核密度增量较大范围示意

Tab.2 Larger increment scope of taxies' OD points by KDE on holidays

\begin{tabular}{cccc}
\hline 节假日核密度增量较大范围示意图 & 核密度分析范围 & 10月 1日 12:00-13:00 & 10月 14日 12:00-13:00 \\
\hline 景点群 & & \\
\hline
\end{tabular}

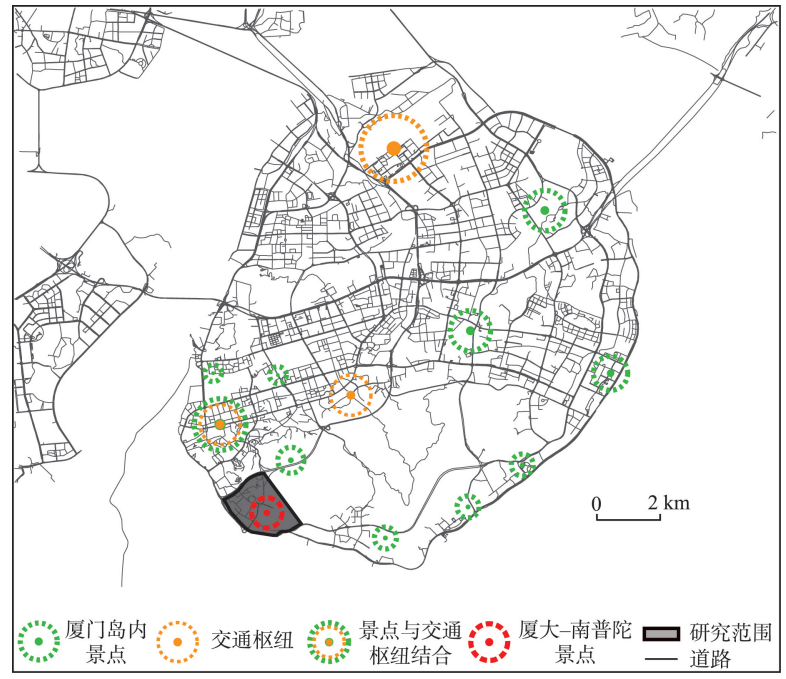

图 4 厦门岛内景点示意图

Fig.4 Location of tourist sites in Xiamen Island
集情况,得到旅游景点周边路网承担车流量的主要 空间分布特征,并进一步对厦大一南普陀寺景点周 边的路网进行分析。

选取研究范围内 2014年 10月 1日 12:00-13:00 时间段的 $\mathrm{FCD}$ 数据, 每隔 $5 \mathrm{~min}$ 对 $\mathrm{FCD}$ 数据进行一 次统计发现(图 6), 出租车载客次数在 60 次上下浮 动, 并没有太大的差距, 说明景点周边路网在该高 峰时间内厦大一南普陀寺景点周围出租车载客次 数持续较为平均。

为了验证该高峰小时内旅游景点周边路网一 直持续高车流量而非瞬时高车流量, 以 $5 \mathrm{~min}$ 为时 间间隔, 将 12:00-13:00 时间段内, 厦大一南普陀寺 景点周边的 FCD 数据分成 12 组进行核密度分析。 经过多次试验, 搜索半径确定为 $400 \mathrm{~m}$ 。分析得到 的核密度最大值出现在 10月 1 日 12:50-12:55, 将该 
时间段的核密度值同样按照自然断裂法分为等级 1 (密度极小)、等级 2 (密度小)、等级 3 (密度较小)、等级 4 (密度较大)、等级 5 (密度大) 等 5 个级别, 进行核密

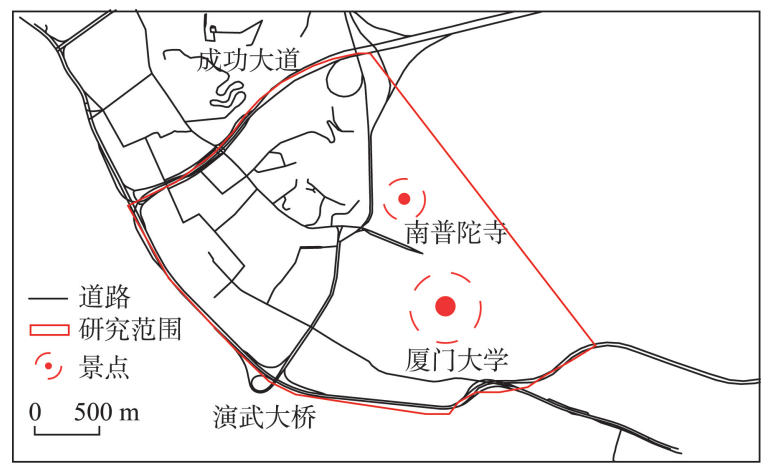

图 5 厦大一南普陀寺景点周边旅游路网研究范围

Fig.5 Research scope of tourism road network around Xiamen University and Nanputuo Temple
度划分。

如图 7 所示, 在研究范围内, 节假日高峰小时 12:00-13:00 的 FCD 核密度持续较高, 高峰小时景点

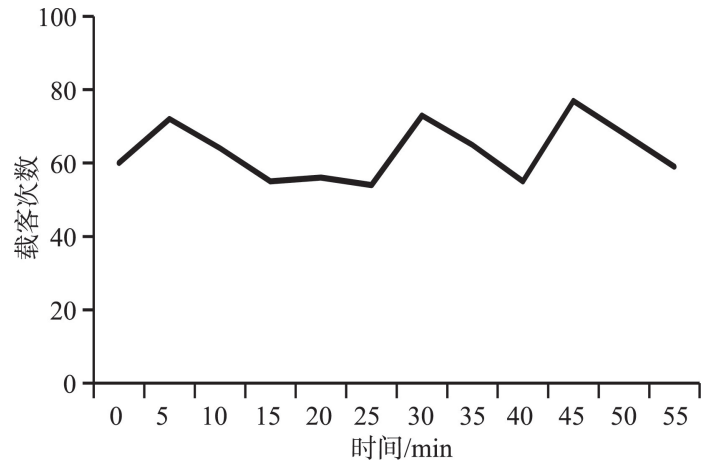

图6 10月 1 日研究范围内出租车载客次数统计

Fig.6 Taxi passenger number statistics in the research area on 1 October
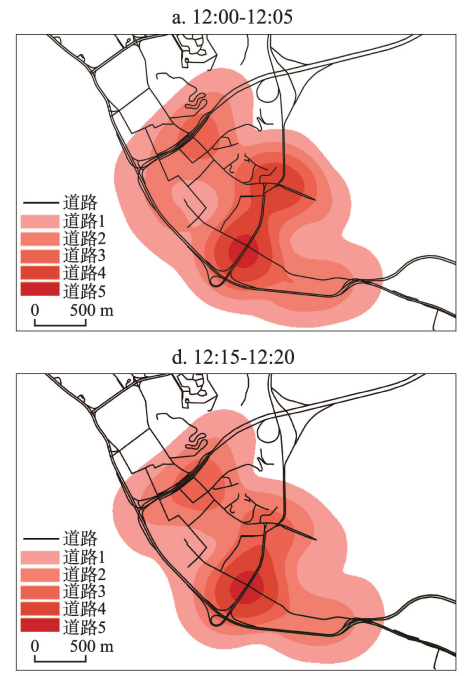

g. 12:30-12:35
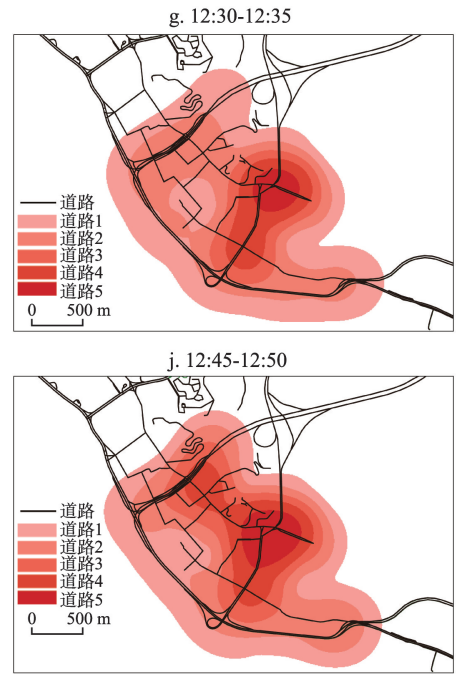

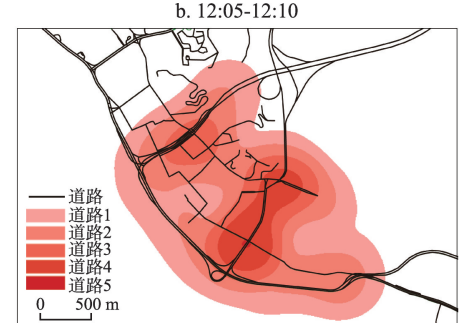

e. $12: 20-12: 25$

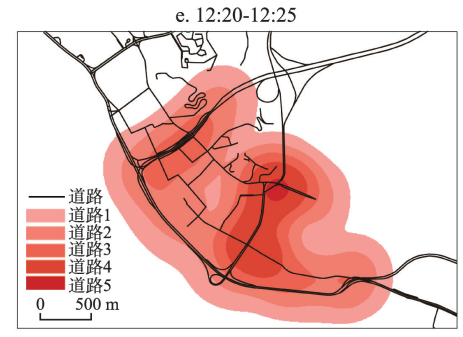

h. $12: 35-12: 40$

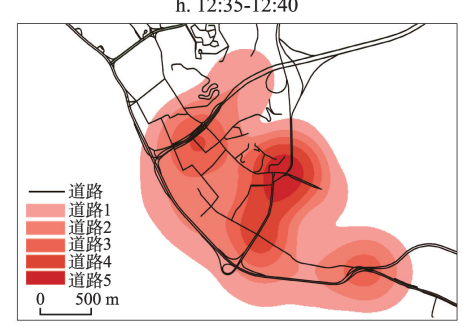

k. 12:50-12:55

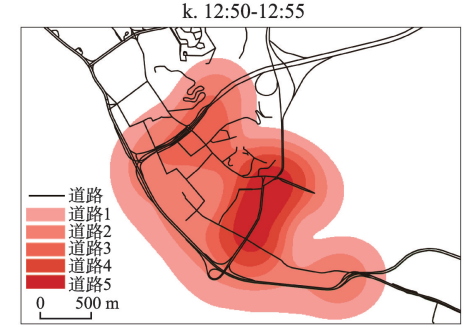

c. $12: 10-12: 15$

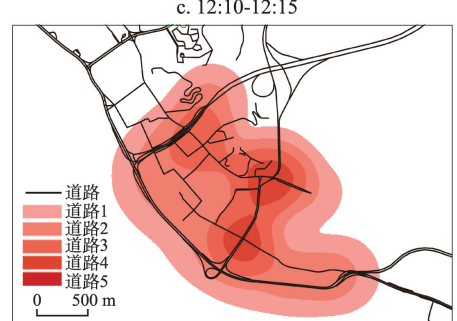

f. $12: 25-12: 30$

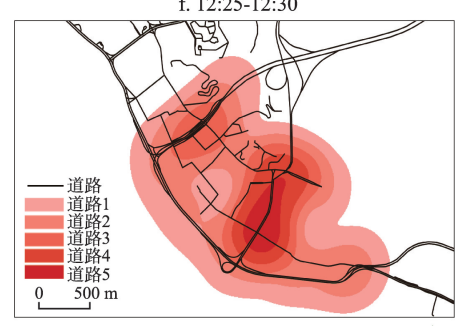

i. $12: 40-12: 45$

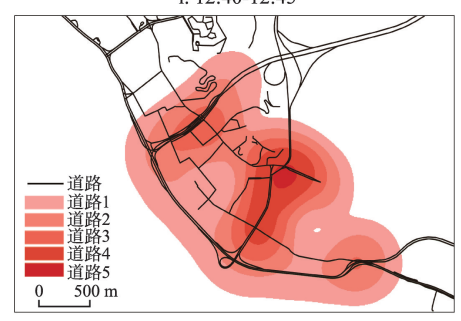

1. $12: 55-13: 00$

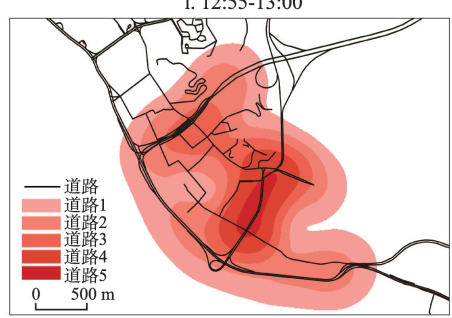

图 7 厦大一南普陀寺景点周围路网 $\mathrm{FCD}$ 核密度分析

Fig.7 KDE of FCD in the road network around Xiamen University and Nanputuo Temple 
周边路网一直存在较大的车流量。同时, 景点周边 $\mathrm{FCD}$ 核密度在高峰小时内持续较大的路段为思明 南路、演武路(思明南路以南路段), 密度等级以景点 为中心向外逐级递减。

\section{3 对比分析}

\subsection{1 对比结果}

基于 FCD 数据的核密度分析, 实际上是对旅游 路网内部各路段所承担的车流量多少进行直观、可 视化的反映, 从而更直接地反映车流在路网中不均 衡的空间分布。将现状车流量与道路设施属性进 行对比, 可发现目前旅游路网发展存在的问题, 为 旅游路网的合理规划与管理提供依据。

对比发现(表 3), 演武路和思明南路 FCD 的最 大核密度等级为 5 ,均为城市双向四车道次干路, 而 城市快速路成功大道和城市主干路环岛路 FCD 的 最高核密度等级只有 3 和 2 。演武路和思明南路等 级与车道数均小于成功大道与环岛路, 但最高核密 度等级却远大于成功大道与环岛路。

由于 FCD 数据的落点分布于每个车道, 进行核 密度分析时无法分辨道路的车道数与宽度。将 FCD 数据根据所落在的路段进行分组, 将每一组数 据根据 $x$ 坐标排序后, 按车道数等间隔删减坐标点, 可得到近似的单车道浮动车分布信息, 再进行核密 度分析, 由此得到路网中各个路段的单车道浮动车 流量核密度分布特征。如图 8 所示, 演武路与思明 南路承担的车流量压力远大于其他路段, 因此可以 推测该景点周边路网中各路段的容量与承担的车 流量之间的严重失衡。

\subsection{2 结果验证}

利用 12:00-13:00 的 FCD 数据对路段速度进行 计算, 以验证以上结论。针对不同道路等级,依据 路段平均速度对路段交通运行通畅程度进行划

\section{表 3 厦大南普陀景点周边路段 FCD 最高核密度 等级与路段基本特征对比}

Tab.3 Highest level of FCD kernel density in the road network around Xiamen University and Nanputuo Temple

compared to the basic characteristics of the roads

\begin{tabular}{lcll}
\hline 道路名称 & 核密度等级 & 道路等级 & 车道数 \\
\hline 成功大道 & 3 & 快速路 & 双向 6车道 \\
环岛路 & 2 & 主干路 & 双向 6 车道 \\
演武路 & 5 & 次干路 & 双向 4 车道 \\
思明南路 & 5 & 次干路 & 双向 4 车道 \\
民族路 & 3 & 支路 & 双向 2 车道 \\
大学路 & 3 & 支路 & 双向 2 车道 \\
\hline
\end{tabular}

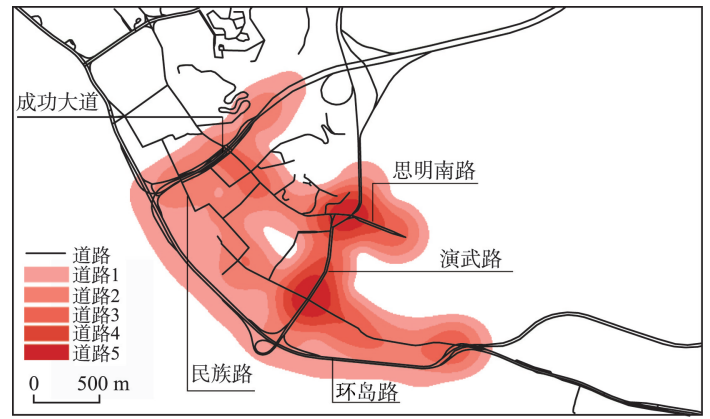

图 8 10月 1日 12:00-13:00 厦大一南普陀寺景点周边 路网单车道FCD核密度分析

Fig.8 KDE of FCD in single lane of the road network around Xiamen University and Nanputuo Temple during 12:00-13:00, 1 October

分。由于未查到相应的国家标准,且厦门市也尚未 出台相关地方标准, 本文采用《北京市道路交通运 行评价指标体系》(北京市质量技术监督局, 2011) 中的划分标准进行分析。将结果匹配至电子地图 发现(图9), 演武路与思明南路靠近厦门大学路段属 于中度拥堵, 环岛路与成功大道则较为畅通, 与上 述核密度分析的研究成果相符。

\section{4 结论与讨论}

出租车作为特殊的公共交通工具, 在旅游节假 日期间承担了大量的游客出行需求。本文选取厦 门市 2014 年 10 月 1 日与 10 月 14 日的 FCD 数据, 首 先判断节假日与工作日由于不同出行目的导致的 出行时空差异, 针对节假日的特殊情况, 对旅游路 网进行出租车 OD 点核密度分析, 最终通过出租车

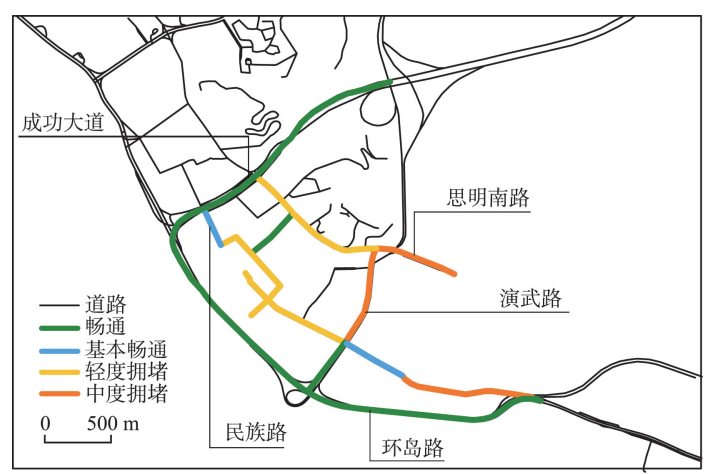

图 910月 1 日 12:00-13:00 厦大一南普陀寺景点 周边路段交通状态示意图

Fig.9 Traffic condition around Xiamen University and Nanputuo Temple during 12:00-13:00, 1 October 
在景点周边路网中的空间分布与路段的等级、车道 数等道路设施属性进行对比分析, 推测路网存在的 问题。主要结论如下:

(1) 旅游交通具有特殊性。通过节假日 (2014 年 10 月 1 日)与工作日(2014年 10 月 14 日)的对比发 现, 出租车出行的高峰时间存在明显差异。从 OD 点核密度分布来看, 在节假日高峰时期, 各个景点 形成了新的较高密度区, 密度等级与范围均大幅度 提升。该结果反映了节假日期间旅游交通的特殊 性, 说明节假日期间旅游交通与工作日通勤交通时 空分布存在差异。

(2) FCD 数据在旅游交通应用的有效性。厦门 市的 FCD 数据具有局限性(只包含出租车数据), 但 由于旅游出行属于弹性出行, 出租车在旅游景点和 旅游基础设施周边存在大量的上下客现象, 本文首 次运用 FCD 数据对节假日期间景点周边交通压力 骤增的路网进行分析研究, 通过对比手段简化了分 析方法, 可较快地找出路网存在的容量问题, 通过 路段速度对其进行验证,证明该方法的有效性。

(3) 厦大一南普陀寺景点周边路网存在问题。 研究发现, 次干路演武路与思明南路为研究区域内 核密度等级最高的路段, 而高等级的环岛路与成功 大道却是区域中核密度等级最低的路段, 区域中的 城市支路核密度等级介于两者之间。通过与路段 等级、车道数等道路设施属性的对比研究推测, 演 武路与思明南路的道路容量在节假日期间无法达 到车流量的要求。

基于节假日期间对出租车的交通需求, 本文通 过出租车在节假日期间旅游景点周边的空间聚集, 在一定程度上反映了旅游景点周边的路网容量, 并 提出相应的改善措施。在厦大一南普陀寺景点的 研究中, 可以打通区域内东西向的内部支路, 或对 较为拥挤的思明南路、演武路进行节假日期间的交 通管控。另外, 也可以通过在节假日期间增开旅游 公交线路,引导游客改变出行方式。但由于数据源 的局限, 对出租车以外的其他交通方式没有考虑。 随着网约车、顺风车等新的出行方式兴起, 人们的 出行习惯正在发生改变。因此, 未来可以通过增加 这类基于定位服务技术所提供的数据源, 对旅游景 点周边的路网容量作进一步深人研究。

\section{参考文献(References)}

北京市质量技术监督局. 2011. DB11/T 785-2011 城市道路
交通运行评价指标体系 [S]. [Beijing Municipal Administration of Quality and Technical Supervision. 2011. DB11/ T 785-2011 urban road traffic performance index[S]].

陈建国, 周素红, 柳林, 等. 2016. 交通拥堵对急救医疗服务 时空可达性的影响: 以广州市为例[J]. 地理科学进展, 35 (4): 431-439. [Chen J G, Zhou S H, Liu L, et al. 2016. Estimating the effect of traffic congestion on accessibility to emergency medical care services: Take Guangzhou as an example[J]. Progress in Geography, 35(4): 431-439.]

陈小鸿, 王翔, 钱昕午. 2013. 城市大型活动车队行驶特征与 交通影响分析 [J]. 同济大学学报: 自然科学版, 41(3): 415-421. [Chen X H, Wang X, Qian X W. 2013. Platoon driving in urban mega- events: Characteristics and traffic impacts[J]. Journal of Tongji University: Natural Science, 41(3): 415-421.]

胡继华, 钟广鹏, 谢海莹. 2012. 基于出租车经验路径的城市 可达性计算方法[J]. 地理科学进展, 31(6): 711-716. [Hu J H, Zhong G P, Xie H Y. 2012. A computational algorithm of urban accessibility based on experiential taxi path[J]. Progress in Geography, 31(6): 711-716.]

冷军强, 张亚平, 韩丽飞, 等. 2010. 冰雪条件下城市路网容 量可靠性 [J]. 哈尔滨工业大学学报, 42(4): 592-596. [Leng J Q, Zhang Y P, Han L F, et al. 2010. Urban road network capacity reliability under ice and snowfall conditions [J]. Journal of Harbin Institute of Technology, 42(4): 592596.]

李晶, 于雷. 2006. 基于 Visum 的路网容量计算理论研究 [J]. 城市交通, 4(2): 68-73. [Li J, Yu L. 2006. A Visum-based method for determining road network capacity[J]. Urban Transport of China, 4(2): 68-73.]

刘伟铭, 田世艳. 2011. 基于地图匹配的城市道路实时交 通状态模糊综合判别方法 [J]. 公路交通科技, 28(10): 86-91. [Liu W M, Tian S Y. 2011. Fuzzy comprehensive identification method of real-time traffic conditions for urban road based on map matching[J]. Journal of Highway and Transportation Research and Development, 28(10): 86-91.]

龙雪琴, 关宏志. 2012. 基于交通安全的城市路网结构优化 方法[J]. 公路交通科技, 29(4): 107-113. [Long X Q, Guan H Z. 2012. Urban road network structure optimization method based on traffic safety[J]. Journal of Highway and Transportation Research and Development, 29(4): 107113.]

马林兵, 魏慧丽, 曹小曙. 2015. 基于 FCD数据的城市有效路 网密度评价: 以广州荔湾区和越秀区为例 [J]. 地理研究, 
ating the effective density of urban road network based on FCD: A case study of Liwan and Yuexiu Districts in Guangzhou[J]. Geographical Research, 34(3): 541-554.] 孙慧娟. 2010. 城市快速路路网布局方法探讨[D]. 西安: 长安 大学. [Sun H J. 2010. Study on the urban expressway transit network planning theory[D]. Xi'an, China: Chang'an University.]

汤国安, 杨昕. 2016. ArcGIS 地理信息系统空间分析实验教 程[M]. 2 版. 北京: 科学出版社. [Tang G A, Yang X. 2016. ArcGIS dili xinxi xitong kongjian fenxi shiyan jiaocheng [M]. 2nd ed. Beijing, China: Science Press.]

王建, 邓卫. 2013. 基于交叉口多相位信号控制的路网容量 [J]. 东南大学学报: 自然科学版, 43(4): 890-894. [Wang J, Deng W. 2013. Capacity of road network with multi-phase signal controlled intersections $[\mathrm{J}]$. Journal of Southeast University: Natural Science Edition, 43(4): 890-894.] 吴健生, 黄力, 刘瑜, 等. 2012. 基于手机基站数据的城市交 通流量模拟[J]. 地理学报, 67(12): 1657-1665. [Wu J S, Huang L, Liu Y, et al. 2012. Traffic flow simulation based on call detail records[J]. Acta Geographica Sinica, 67(12): 1657-1665.]

张伟. 2014. 大型公共场所行人交通状态评价及其应急疏散 方法研究[D]. 长春: 吉林大学. [Zhang W. 2014. Research on methods of pedestrian traffic condition evaluation and emergency evacuation at large public venues[D]. Changchun, China: Jilin University.]

Jenelius E. 2009. Network structure and travel patterns: Explaining the geographical disparities of road network vulnerability[J]. Journal of Transport Geography, 17(3): 234244.

Li Q Q, Zhang T, Wang H D, et al. 2011. Dynamic accessibility mapping using floating car data: A network-constrained density estimation approach[J]. Journal of Transport Geography, 19(3): 379-393.

Shi X. 2010. Selection of bandwidth type and adjustment side in kernel density estimation over inhomogeneous backgrounds[J]. International Journal of Geographical Information Science, 24(5): 643-660.

\title{
Road network capacity of tourist site's periphery based on FCD: Taking Xiamen Island as an example
}

\author{
GAO Yueer, CHEN Shuting, ZHENG Chengyu, BIAN Jingwei
}

(School of Architecture, Huaqiao University, Xiamen 361021, Fujian, China)

\begin{abstract}
With the rapid development of tourism, there has been a sharp increase in traffic demand in tourist cities during public holidays, which results in various traffic problems. This article examines the traffic characteristics of Xiamen using floating car data (FCD) on 1 October (a public holiday) and 14 October 2014 (a normal working day). The spatial scope of the study was determined by the spatiotemporal differences between the number of trips on the public holiday and the normal working day using the origin and destination's kernel density estimation method. Road network capacity was estimated by analyzing the change of traffic volume near the tourist sites using kernel density estimation based on FCD, and comparing with road facility attributes such as the grade of the road section, and the number of lanes. Finally, the study validates the feasibility of the method by estimating the traffic speed of the road sections in the road network. The results show that there exist spatiotemporal differences between the tourist traffic on the public holiday and commuting traffic on the normal working day, and the method for road network capacity study near the tourist sites is effective based on FCD. The road capacity near Xiamen University and Nanputuo Temple cannot meet the traffic demand during the public holiday. The estimation of road network capacity near tourist sites can provide a basis for reasonable planning and management of the tourism road network during the tourist season.
\end{abstract}

Key words: tourist traffic; road network capacity; kernel density estimation; floating car data (FCD); Xiamen Island 